\title{
MICROPHONICS DETUNING IN THE 500 MHz SUPERCONDUCTING CESR CAVITIES*
}

\author{
M. Liepe ${ }^{\dagger}$ and S. Belomestnykh, Cornell University, Ithaca, NY 14853, USA
}

\section{Abstract}

The RF system of the Cornell Electron Storage Ring (CESR) consists of four superconducting (s.c.) $500 \mathrm{MHz}$ cavities. For a charm-tau upgrade (CESR-c, see [1]) we plan to drive two cavities by one klystron and to operate some cavities in passive mode [2].

During the previous high energy run the RF system performance was not significantly affected by microphonic cavity detuning, since the cavities were operated under strong beam loading with a low loaded quality factor of $2 \cdot 10^{5}$. However, in low energy CESR-c operation with increased loaded Q-factor the RF system becomes less tolerant to microphonics, especially when two cavities are driven by one klystron. In order to address this potential problem we have studied microphonics in the CESR cavities in detail. Significant improvements have been achieved by reducing major contributions to the detuning. For further improvements we plan to study the performance of an active microphonics compensation scheme, which is based on a fast piezoelectric-driven frequency tuner.

\section{INTRODUCTION}

Environmental microphonic noise can cause fluctuation of the cavity resonance and as a result produce amplitude and phase modulations of the cavity field, which then can affect beam quality or RF system performance. This is especially true for high-Q superconducting cavities. Though this noise was an issue when the very first s.c. cavity has been installed in CESR [3], it was drastically reduced later and is not considered a limiting factor in the present configuration of the CESR RF system with external cavity quality factors of approximately $2 \cdot 10^{5}$.

However, as CESR is being modified for operating at lower energies, there will be changes in the RF system parameters and configuration [4]. First of all, two out of four s.c. cavities will be operated in a passive mode (i.e. driven only by the beam current) at high voltage and external quality factor [2]. Second of all, the remaining two active cavities will have to operate at significantly higher $Q_{e x t}\left(6 \cdot 10^{5}\right.$ to $1.2 \cdot 10^{6}$ ) than at present. Hence we need to reassess the influence of microphonics.

Microphonics influence on the beam quality via the active cavities should be negligible as RF feedback loops are taking care of the cavity field stability. The issue here is the RF system performance. Bigger RF power overhead is required to compensate for the microphonics detuning. Suppose the cavity is tuned to compensate the reactive

\footnotetext{
* Work is supported by the National Science Foundation.

†mul2@cornell.edu
}

beam loading, then the required forward power $P_{f}$ from the klystron is

$$
P_{f}=\frac{V_{c}^{2}}{4 \frac{R}{Q} Q_{e x t}}\left\{\left[1+\frac{I_{b} \frac{R}{Q} Q_{e x t}}{V_{c}} \cos \phi_{s}\right]^{2}+\left[\frac{2 Q_{e x t} \delta \omega_{m}}{\omega}\right]^{2}\right\}
$$

Here $V_{c}$ is the cavity voltage, $I_{b}$ is the average beam current, $\phi_{s}$ is the synchronous phase, $\delta \omega_{m}$ is the amplitude of the microphonic noise, and $\omega$ is the RF frequency. At full beam loading (i.e. matching conditions) the relative contribution of the microphonics to the power demands is then

$$
\frac{\delta P_{f}}{P_{f}}=\left(\frac{Q_{e x t} \delta \omega_{m}}{\omega}\right)^{2}
$$

Accordingly $42 \mathrm{~Hz}$ microphonics at the external Q of $1.2 \cdot 10^{6}$ results in $1 \%$ of forward power fluctuation. Since a fluctuation of a few percent can be tolerated, keeping the peak microphonic detuning below $100 \mathrm{~Hz}$ is desirable for the CESR-c cavities.

In the passive cavities microphonic noise will cause amplitude and phase modulation of the cavity voltage [5], which in turn will affect the bunch length. While phase modulation for the CESR-c conditions is reasonably small, the voltage amplitude modulation can be significant. In passive operation the cavities are detuned by several bandwidths ( $\Delta \omega \gg$ cavity bandwidth) [2], and for this condition the field amplitude modulation due to microphonics is given by

$$
\left|\frac{\delta V_{c}}{V_{c}}\right| \approx \frac{2 V_{c}}{I_{b} \frac{R}{Q}} \frac{\delta \omega_{m}}{\omega} .
$$

We find that a microphonics amplitude of $30 \mathrm{~Hz}$ gives a $1 \%$ voltage amplitude fluctuation. A fluctuation of a few percent can be tolerated with respect to the resulting bunch length modulation. Accordingly keeping the peak microphonic detuning below $100 \mathrm{~Hz}$ is desirable also for the passive cavities in CESR-c.

\section{MICROPHONICS STUDIES AND REDUCTION}

Since microphonic cavity detunig is a potential limiting factor during CESR-c operation, we started a measurement program to study the microphonics levels in the CESR cavities in detail. A typical measured fluctuation of the 500 $\mathrm{MHz}$ mode frequency of a CESR cavity (cavity E2) is shown in Figure 1. We identified two significant contributions to the measured fluctuation of the accelerating mode frequencies:

- The slow frequency tuner excites vibration of the cavity. The tuner is driven by a stepping motor and is 
used to compensate slow drifts of the cavity eigen frequency. A sequence of steps of the stepping motor has a step frequency of several $10 \mathrm{~Hz}$. If this frequency matches a mechanical resonance of the cavity-cryostat system, strong mechanical vibrations can be excited, which then can result in a modulation of the $500 \mathrm{MHz}$ electrical resonance frequency; see Figure 1 and Figure 2. In the example shown, a mechanical resonance at about $75 \mathrm{~Hz}$ is excited by a sequence of motor steps; see also Figure 6.

- The second contribution to the measured cavity microphonics is not due to vibration of the cavity itself, but results from a phase modulation of the $500 \mathrm{MHz}$ CESR reference signal; see Figure 3. Cleary visible are sidebands at frequencies $f=500 \mathrm{MHz} \pm n \cdot 120 \mathrm{~Hz}$. Since the RF feedback loops measure the phase of the RF field with respect to the $500 \mathrm{MHz}$ reference signal, it appears as if the $500 \mathrm{MHz}$ electrical cavity resonance frequency is fluctuating with frequencies $f=n \cdot 120 \mathrm{~Hz}$; see Figure 2 .
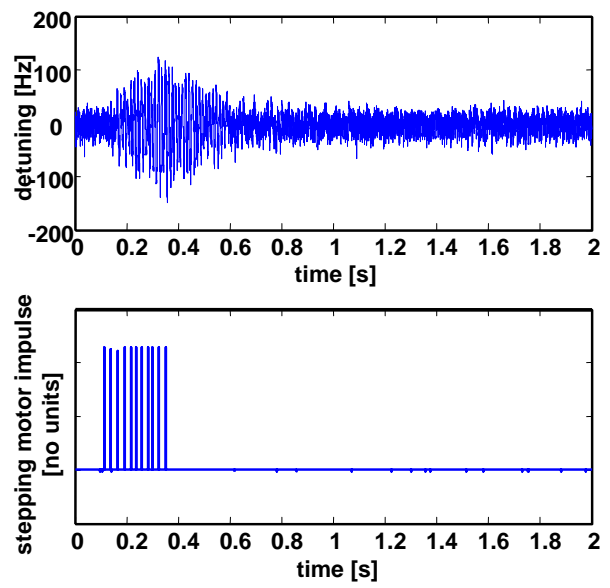

Figure 1: Top: Fluctuation of the $500 \mathrm{MHz}$ mode frequency of cavity E2 before improvements have been made. The peak cavity detunig is $150 \mathrm{~Hz}$, the fluctuation is 27 $\mathrm{Hz}$ rms. Bottom: Stepping motor activity of the slow frequency tuner during the same time interval. Each impulse marks a motor step.

The microphonics measurements showed rms amplitudes of 20 to $30 \mathrm{~Hz}$ and peak microphonics amplitudes of about $150 \mathrm{~Hz}$ for the four CESR cavities. The peak values are above our $100 \mathrm{~Hz}$ goal for the low energy CESR-c operation, and a reduction of the microphonics levels was needed. Since we had identified two major contributions to the cavity microphonics, a logical step was to reduce these sources. To avoid vibration excitation by the slow frequency tuner the step sizes of the motor have been reduced. As a result of this, the cavity is now tuned smother with a faster sequence of smaller steps, and the excitation of mechanical resonances is reduced significantly; see Figure 4.
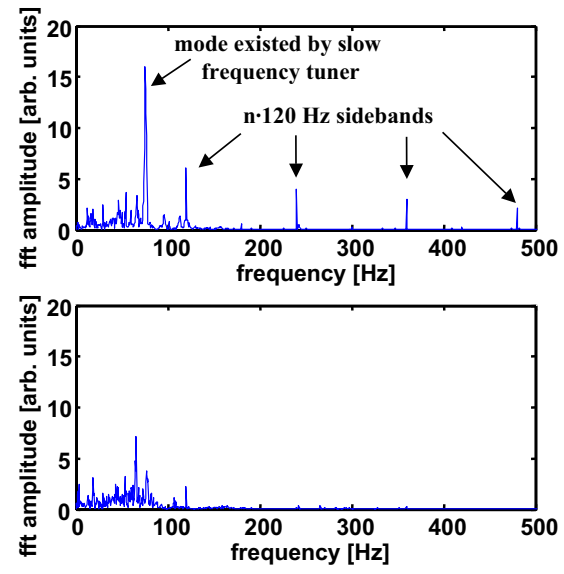

Figure 2: Measured microphonics spectrum. Top: Fourier transform of the cavity detuning signal shown in Figure 1 (before improvements have been made). Bottom: Fourier transform of the cavity detuning signal shown in Figure 4 (after improvements have been made).

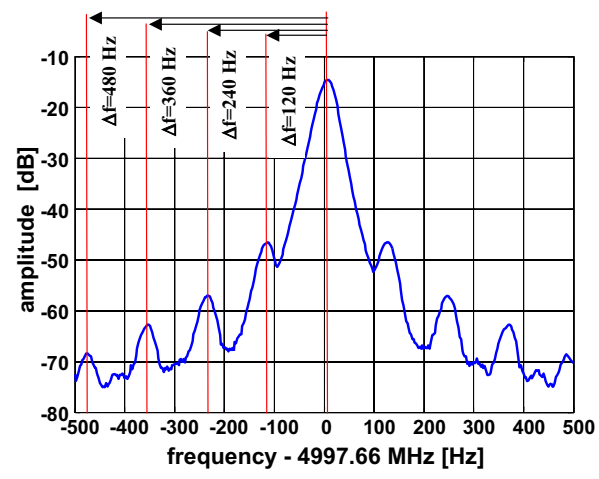

Figure 3: Spectrum of the $500 \mathrm{MHz}$ CESR reference signal before improvements have been made.

The sidebands of the $500 \mathrm{MHz}$ reference signal were eliminated by improving the grounding of the RF signal generation hardware; see Figure 2. Due to these steps the microphonics levels in the CESR cavities were reduced by about a factor of two. Typical rms values are now $15 \mathrm{~Hz}$ with peak microphonics amplitudes of about $60 \mathrm{~Hz}$, i.e. well below the CESR-c requirements.

\section{FUTURE PLANS}

After eliminating major sources of the microphonic cavity detuning, further reduction of the frequency fluctuation can be achieved by using a fast active compensation scheme. Piezo-electric elements are frequently used for vibration damping, and have been used to drive a fast cavity frequency tuner [6]. To investigate a fast frequency tuning scheme for the CESR cavities, piezo-elements have been integrated into the tuning frame of the slow steppingmotor driven frequency tuner; see Figure 5. In order to design a feedback loop for fast detuning compensation, it 

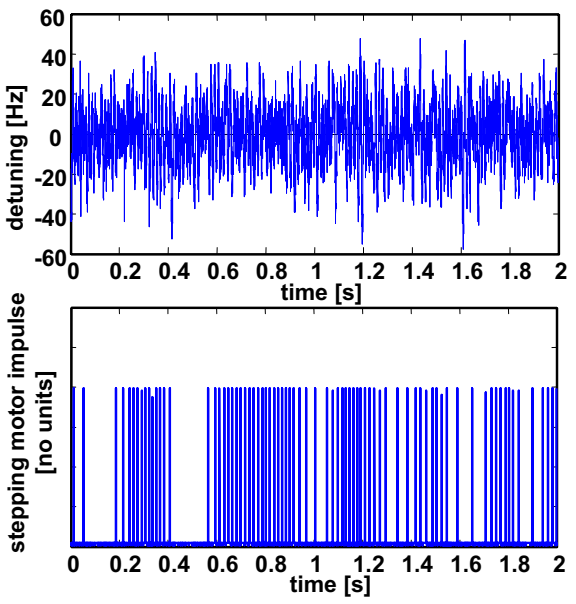

Figure 4: Top: Fluctuation of the $500 \mathrm{MHz}$ mode frequency of cavity E2 after improvements have been made. The peak cavity detuning is $60 \mathrm{~Hz}$, the fluctuation is $14 \mathrm{~Hz}$ rms. Bottom: Stepping motor activity of the slow frequency tuner during the same time interval. Each impulse marks a motor step.

is essential to identify the dynamics of the system. Figure 6 shows the measured transfer function from the piezo drive signal to the cavity detuning measured at cavity E2. Clearly visible are mechanical resonances above $40 \mathrm{~Hz}$, with the strongest resonance at about $75 \mathrm{~Hz}$. Note that the $75 \mathrm{~Hz}$ resonance is excited by the steps of the slow frequency tuner during the microphonics measurement shown in Figure 1 and 2. Because of the low frequency resonances feedback control will be challenging and a complex control algorithm will be required. A digital control system is presently under development [7] and will provide the needed computational capacities for a digital controller. Once the hardware is finished we plan to study the performance of a fast microphonics compensation controller. Beside a further reduction of the microphonic cavity detuning at the CESR cavities, we hope to benefit from this experience when designing a fast frequency tuner for the cavities of the proposed Cornell ERL prototype [8, 9], where low microphonics amplitudes are essential [10].

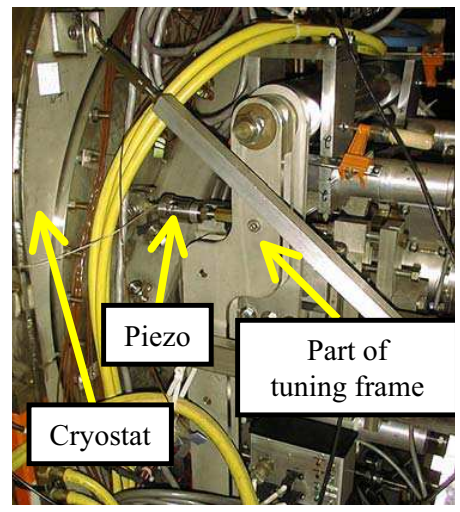

Figure 5: CESR cryostat with piezo frequency tuner.
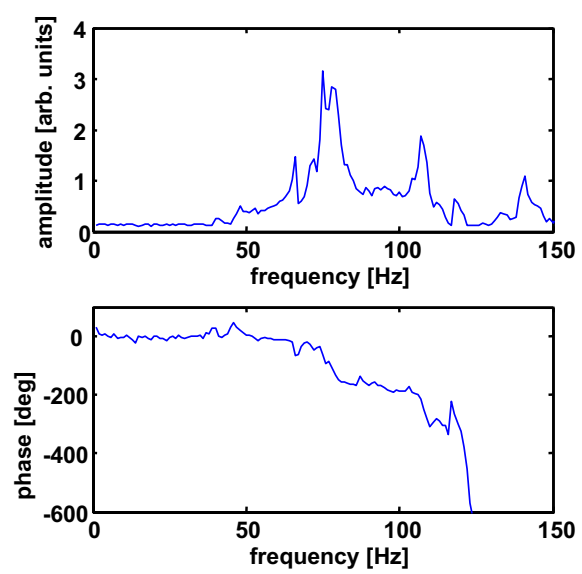

Figure 6: Transfer function from the piezo drive signal to the cavity detuning measured at cavity E2. Top: amplitude. Visible are mechanical resonances above $40 \mathrm{~Hz}$. Bottom: phase.

\section{CONCLUSIONS}

For the low energy CESR-c operation microphonics amplitudes in the four s.c. cavities below $100 \mathrm{~Hz}$ are desirable. Detailed studies of the microphonics levels and frequency spectrum enabled us to identify major contributions to the frequency fluctuation. Eliminating these sources reduced the microphonics amplitudes by a factor of two to a level well below the CESR-c requirements. Further reduction appears feasible by using a fast active detuning compensation scheme based on a piezo-electric driven frequency tuner.

\section{ACKNOWLEDGEMENTS}

We would like to thank J. Dobbins, R. Kaplan, J. Sears and V. Veshcherevich for their help.

\section{REFERENCES}

[1] D. Rice, Proceedings of the European Particle Accelerator Conference 2002, Paris, June 2002, p. 428.

[2] S. Belomestnykh et al., this conference, paper TPAB048.

[3] S. Belomestnykh et al., Proceedings of the 2001 Particle Accelerator Conference, Chicago, IL, June 2001, p. 1065.

[4] S. Belomestnykh, Report SRF020918-06, Cornell Laboratory for Elementary-Particle Physics (2002).

[5] S. Belomestnykh, et al., Proceedings of the 2001 Particle Accelerator Conference, Chicago, IL, June 2001, p. 1062.

[6] M. Liepe et al., Proceedings of the 2001 Particle Accelerator Conference, Chicago, June 2001, p. 1074.

[7] M. Liepe et al., this conference, paper FPAB027.

[8] Study for a proposed Phase I ERL Synchrotron Light Source at Cornell University, ed. by S. Gruner and M. Tigner, CHESS Tech. Memo 01-003, JLAB-ACT-01-04 (July 2001)

[9] C. Sinclair et al., this conference, paper TOAC005.

[10] M. Liepe et al., this conference, paper TPAB056. 